\title{
Metamaterial/graphene active terahertz modulators
}

\author{
Riccardo Degl'Innocenti ${ }^{1}$, Stephen J. Kindness ${ }^{2}$, Nikita W. Almond ${ }^{2}$, Wladislaw Michailow ${ }^{2}$, Philipp Braeun- \\ inger-Weimer ${ }^{3}$, Stephan Hofmann ${ }^{3}$, Harvey E. Beere ${ }^{2}$, David A. Ritchie ${ }^{2}$ \\ ${ }^{1}$ Department of Engineering, University of Lancaster, Bailrigg, Lancaster, LA1 4YW, UK \\ ${ }^{2}$ Cavendish Laboratory, University of Cambridge, J. J. Thomson Avenue, Cambridge, CB3 \\ OHE, UK \\ ${ }^{3}$ Department of Engineering, University of Cambridge, 9 J. J. Thomson Avenue, Cambridge,
}

CB3 0FA, UK

\begin{abstract}
Within the last years there has been a tremendous thrust into research and technology in the $\mathrm{THz}$ spectral region (broadly defined as 0.1-10 $\mathrm{THz}$ ) mainly driven by the unique potential where this radiation finds applications in, such as imaging, spectroscopy and communication. In all these fields a fast, integrated and versatile platform for modulating light is required. Metamaterial/graphene devices fulfill all these requirements as their subwavelength nature lends itself naturally to strong light-matter interaction, and therefore highly efficient and miniaturized devices. Graphene's unique properties, e.g. the large carrier concentration modulation, provide a large degree of compatibility with several architectures which can be exploited in a range of modulation or detection schemes. Finally, metamaterial/graphene devices realize a fast, versatile platform, which can be easily scaled to other frequencies, and adapted into amplitude, frequency, polarization and phase modulators, as well as integrated detectors, for the next generation of wirelesscommunication.
\end{abstract}

Index Terms - terahertz metamaterials, amplitude modulation, frequency modulation, active filters, quantum cascade lasers.

\section{INTRODUCTION}

Active terahertz metamaterial and plasmonic devices have helped to unlock a myriad of applications, ranging from spectroscopy and imaging to communications. The interest in the $\mathrm{THz}$ communications sector [1] stems, amongst others, from the high carrier frequency and large bandwidth which allows high data transfer [2]. Metamaterials, subwavelength resonant features, are designed to exhibit an artificial electro-magnetic response to the incident light and are of particular interest in the terahertz frequency range, because of the intrinsic difficulties inherent to the realization of efficient devices in this spectral region lying between the electronic and photonic range. They have been reported in a variety of configurations, normally arranged into arrays. Furthermore, they have been shown to be a promising route in combination with chemical-vapor deposited (CVD) graphene, for the realization of the next generation of efficient, highspeed, amplitude, frequency, phase and polarization modulation of $\mathrm{THz}$ radiation [3] and to be compatible with detectors possessing similar footprints/performance [4,5]. In particular, broadband, high modulation depth [6] amplitude modulators have been reported, and a few groups demonstrated reconfiguration speed in excess of $100 \mathrm{MHz}$ operation at frequencies above $1 \mathrm{THz}[7,8]$. Continuous active frequency modulators were recently achieved by integrating metal-coupled resonator arrays with electrically tunable graphene and reporting tunable band pass/reject filters Fig. 1.

Scanning electron microscope picture of the basic metamaterial/graphene unit for the polarization modulator device. The incident light is polarized along y direction and excites the bright resonator element. The two dark resonators are indirectly excited by the bright resonator and re-irradiate a component of the E-field along the $\mathrm{x}$ direction. By electrostatically biasing the graphene areas, the polarization of the incoming radiation is continuously tuned. with a tuning range of $\sim 100 \mathrm{GHz}$ operating at $1.5 \mathrm{THz}$ [9]. A similar architecture has been extended to achieve active polarization modulators, since these can be used in $\mathrm{THz}$ communication protocols, such as with polarization shift keying and polarization division multiplexing [10].
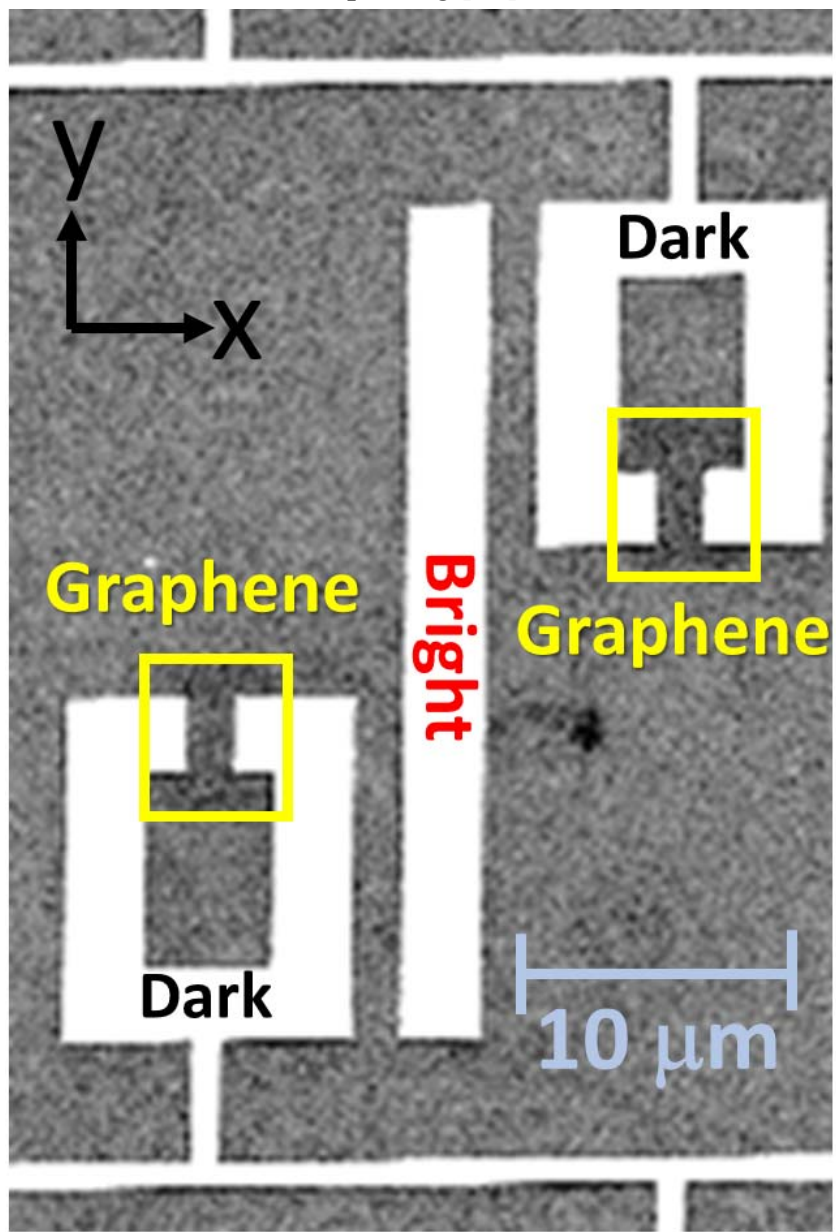

Fig. 1. Scanning electron microscope picture of the basic metamaterial/graphene unit for the polarization modulator device. The incident light is polarized along y direction and excites the bright resonator element. The two dark resonators are indirectly excited by the bright resonator and re-irradiate a component of the E-field along the $\mathrm{x}$ direction. By electrostatically biasing the graphene areas, the polarization of the incoming radiation is continuously tuned. 


\section{BASIC OPERATING PRINCIPLES}

The metamaterial device exploits coupled plasmonic resonators to exhibit an electromagnetically induced transparency analog, resulting in the splitting of the resonance into coupled hybrid optical modes. The bright resonator, directly excited by the incident light oriented along the main y-axis is capacitively coupled to the dark lateral resonators, shunted by graphene in the gaps, as showed in Fig. 1. These resonators exhibit an indirectly excited resonant response providing an oscillating electric dipole component along the $\mathrm{x}$ direction. Depending on the selected frequency range this device provides optical activity or circular dichroism. The metallic ( $\mathrm{Ti} / \mathrm{Au}$, typically $15 / 100 \mathrm{~nm}$ ) features are designed via e-beam lithography and thermal evaporation on top of standard $\mathrm{SiO}_{2} / \mathrm{p}$-doped Boron doped $\mathrm{Si}$ substrate $\left(300 \mathrm{~nm} \mathrm{SiO}_{2}\right.$ on $\sim 0.5 \mathrm{~mm} \mathrm{Si} \mathrm{substrate)}$ and arranged into arrays with typical footprints of $\sim 1-2 \mathrm{~mm}^{2}$. Graphene is transferred onto the metamaterial arrays in a second processing step and typically defined into patches using a second electron beam lithographic step followed by oxygen plasma. Graphene's Dirac point is normally found at positive voltages, compatible with p-doped CVD graphene at biases between $30 \mathrm{~V}$ and $100 \mathrm{~V}$ for exposed graphene, and at voltages typically below $10 \mathrm{~V}$ when encapsulated with a dielectric layer. By electrostatically changing the graphene Fermi energy via the back-gate, the coupling between the two elements is continuously modified thus tuning the transmitted polarization properties.

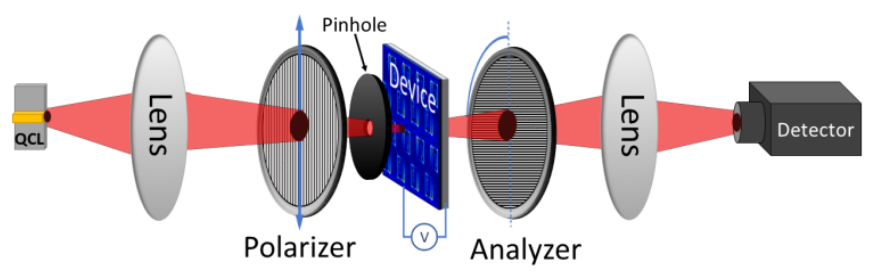

Fig. 2. Typical schematic arrangement used for the characterization of metamaterial/graphene devices with QCLs.

These non-optimized devices are capable to achieve an allelectronic continuous tuning of the polarization plane angle, reaching a modulation range $>20^{\circ}$ around $1.75 \mathrm{THz}$. The determination of the reconfiguration speed for amplitude, frequency and polarization modulators and detectors, is performed by using terahertz quantum cascade lasers (QCLs). These unipolar, frequency narrow and powerful, semiconductor lasers are ideal sources because of their high stability. The scheme adopted for the speed measurements does not require any cryogenic fast detector but is compatible with slowresponse devices such as Golay cells, as it is reported in detail in $[8,11]$. The experimental arrangement used for these measurements and the standard modulator characterization with QCLs is showed in Fig. 2. The radiation emitted from a QCL is collimated and focused onto the sample array by using lenses with focal length of $\sim 2.5-5 \mathrm{~cm}$. A pin-hole is placed in closed proximity to the sample's surface to ensure that the radiation is impinging only on the metamaterial defined area. The output radiation is collimated and focused onto the $\mathrm{THz}$ Golay detector. Grid polarizers are inserted to ensure a precise polarization control of the input/output radiation and to probe the output radiation polarization. In the case of the polarization devices as showed in Fig. 1, the analyzing polarizer can be oriented along the input $y$-direction, to select the $E_{y}$ component of the electric field, or crossed to retrieve the induced $\mathrm{E}_{\mathrm{x}}$ component.

\section{RESULTS}
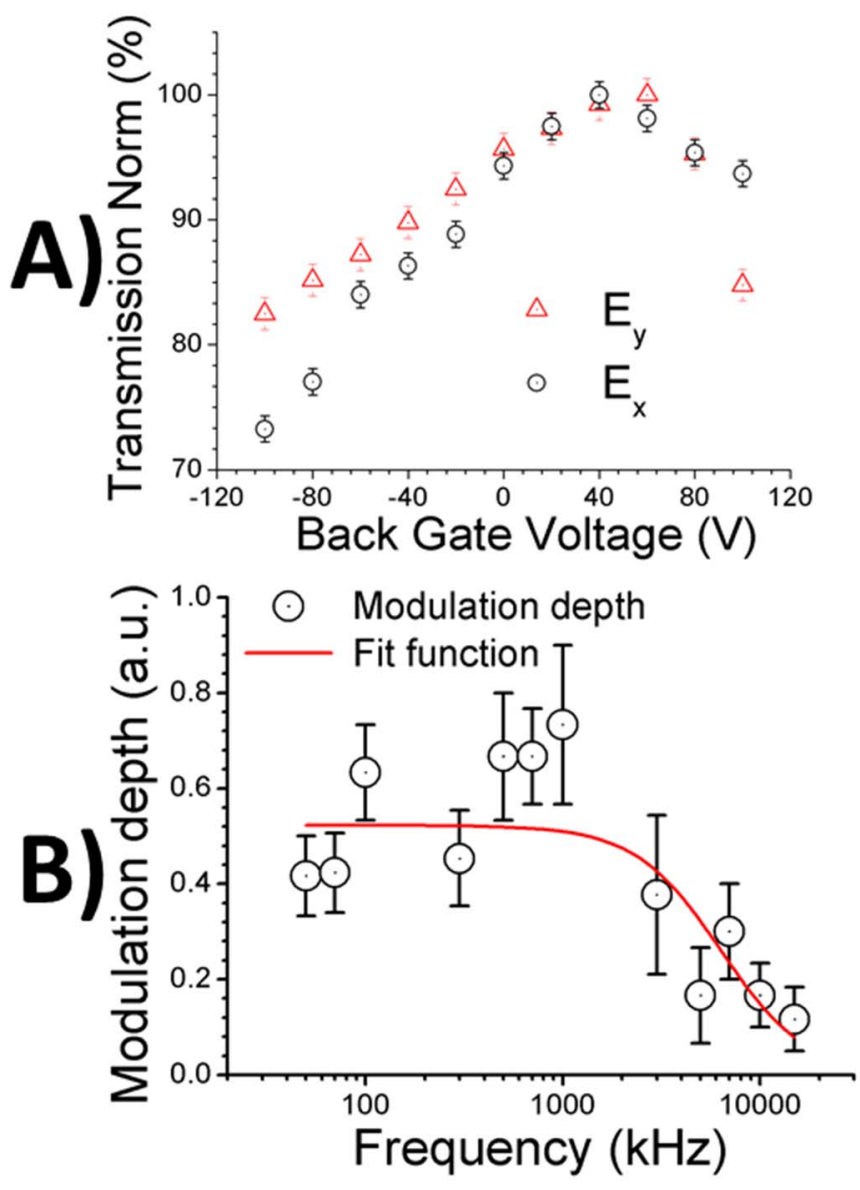

Fig. 3. A) Transmission data acquired at different back-gate voltages, with the analyzer placed parallel to the incident radiation (Ey) and perpendicular (Ex). B) Speed measurements performed with the QCL. The data were fitted with a standard low-pass filter function

Typical transmission measurements recorded with a QCL operating in single emission frequency at $1.94 \mathrm{THz}$ are reported in Fig. 3 a). Both components increased at this frequency by increasing the applied voltage towards the Dirac point. From these measurements it is possible to infer a Dirac point position approximately at $\sim 40 \mathrm{~V}$, in good agreement with the $\sim 30$ $\mathrm{V}$ measured for a large uniform graphene area transferred and processed together with the metamaterial areas. Fig. 3 b) shows the reconfiguration speed measurements; the QCL was 
operated with a repetition rate of $620 \mathrm{~Hz}$, a duty cycle of $10 \%$ and a gate frequency of $12 \mathrm{~Hz}$ detected by the Golay cell. A fast, $10 \mathrm{~V}$ peak-to-peak frequency modulation was fed to the device through the back gate. A first approximate fit of the experimental data performed by using the standard formula for the RC low pass filter, yields a maximum modulation speed of $6.3 \pm 1.5 \mathrm{MHz}$, in good agreement with the values reported in literature [11]. Practically, the insertion of an output polarizer also allows the utilization of these polarization modulators as amplitude modulators as well. Differently from the previously reported experiments, also a component of the E-field perpendicular to the incident radiation can be modulated. The modulation depths recorded were $\sim 18 \%$ and $\sim 28 \%$ for the $\mathrm{E}_{\mathrm{y}}$ and $\mathrm{E}_{\mathrm{x}}$ components, respectively. It is envisaged that configuration speeds in excess of $100 \mathrm{MHz}$ and higher amplitude modulation depths could be attained by optimizing the device architecture

\section{CONCLUSION}

In conclusion metamaterial/graphene devices are a viable route for the realization of an efficient $\mathrm{THz}$ integrated platform. Due to its versatility, this approach can be implemented in a variety of configurations from amplitude to polarization modulators and detectors. This work establishes the basis for the realization of novel $\mathrm{THz}$ wireless communication protocols for high-speed data transfer.

\section{ACKNOWLEDGEMENT}

R.D. acknowledges support from the Engineering and Physical Sciences Research (EPSRC) Council (Grant No EP/S019383/1) and from the Royal Society (RSG $\backslash R 1 \backslash 180148$ - Research Grant). S.H. and P.B.-W. acknowledge funding from EPSRC (EP/K016636/1). S.J.K., N.W.A., W.M., H.E.B., D.A.R., acknowledge financial support from the EPSRC (Grant No. EP/P021859/1, Hyper Terahertz).

\section{REFERENCES}

[1] T. Nagatsuma, G. Ducournau, C. C. Renaud, "Advances in terahertz communications accelerated by photonics" Nature Photonics, vol. 10, pp 371-379, 2016.
[2] X. Yu1, S. Jia, H. Hu, M. Galili, T. Morioka, P. U. Jepsen, and L. K. Oxenløwe " $160 \mathrm{Gbit} / \mathrm{s}$ photonics wireless transmission in the $300-500$ GHz band" APL Photon., vol 1, 081301, 2016.

[3] R. Degl'Innocenti, S. J. Kindness, H. E. Beere, D. A. Ritchie "Allintegrated terahertz modulators" Nanophotonics, vol. 7, pp 127-144, 2018.

[4] R. Degl'Innocenti, L. Xiao, D. S. Jessop, S. J. Kindness, Y. Ren, H. Lin, J. A. Zeitler, J. A. Alexander-Webber, H. J. Joyce, P. BraeuningerWeimer, S. Hofmann, H. E. Beere and D. A. Ritchie "Fast room temperature detection of terahertz quantum cascade lasers with graphene loaded bow-tie plasmonic antenna arrays" ACS Photonics, vol. 3, pp 1747$1753,2016$.

[5] R. Degl'Innocenti, L. Xiao, S.J. Kindness, V. S. Kamboj, B. Wei, P. Braeuninger-Weimer, K. Nakanishi, A. I. Aria, S. Hofmann, H. E. Beere, and D. A. Ritchie, "Bolometric detection of terahertz quantum cascade lasers with graphene-plasmonic antenna arrays" Journal of Physics D, vol. 50, 174001, 2017.

[6] Y. Sun, R. Degl'Innocenti, D. A. Ritchie, H. E.Beere, L. Xiao, M. Ruggiero, J. A. Zeitler, R. I. Stantchev, D. Chen, Z. Peng, E. Macpherson, $\mathrm{X}$. Liu, "Graphene-loaded metal wire grating for deep and broadband THz modulation in total internal reflection geometry" Photonics Research, vol. 6, pp 1151-1157, 2018.

[7] D. S. Jessop, S. J. Kindness, L. Xiao, P. Braeuninger-Weimer, H. Lin, Y. Ren, C. X. Ren, S. Hofmann, J. A. Zeitler, H. E. Beere, D. A. Ritchie and R. Degl'Innocenti, "Graphene based plasmonic terahertz amplitude modulator operating above $100 \mathrm{MHz}$ ", Appl. Phys Lett., vol. 108, 171101, 2016.

[8] G. Liang, X. Hu, X. Yu, Y. Shen, L. H. Li, A. G. Davies, E. H. Linfield, H. K. Liang, Y. Zhang, S. F. Yu, and Q. J. Wang, "Integrated Terahertz Graphene Modulator with 100\% Modulation Depth". ACS Photonics, vol. 2, pp 1559-1566, 2015.

[9] S. J. Kindness, N. W. Almond, B. Wei, R. Wallis, W. Michailow, V. S. Kamboj, P. Braeuninger-Weimer, S. Hofmann, H. E. Beere, D. A. Ritchie, R. Degl'Innocenti, "Active control of electromagnetically induced transparency in a coupled plasmonic resonator array with graphene for continuous frequency control of terahertz radiation" $A d v$. $O p$ tical Mater. vol 6, 1800570, 2018.

[10] N. Oshima, K. Hashimoto, S. Suzuki, M. Asada, "Terahertz Wireless Data Transmission With Frequency and Polarization Division Multiplexing Using Resonant- Tunneling-Diode Oscillators" IEEE Transactions on Terahertz Science and Technology, vol. 7, pp. 593-598, 2017.

[11] R. Degl'Innocenti, D. S. Jessop, C. W. O. Sol, L. Xiao, S. J. Kindness, H. Lin, J. A. Zeitler, P. Braeuninger-Weimer, S. Hofmann, Y. Ren, V. S. Kamboj, J. Griffiths, H. E. Beere and D. A. Ritchie "Fast modulation of terahertz quantum cascade lasers using graphene loaded plasmonic antennas" ACS Photonics, vol. 3, pp 464-470, 2016. 